\title{
ARTICLES \\ MODERN PORTFOLIO THEORY: SOME MAIN RESULTS
}

\author{
By HEINZ H. MülLER
}

University of Zürich

\begin{abstract}
This article summarizes some main results in modern portfolio theory. First, the Markowitz approach is presented. Then the capital asset pricing model is derived and its empirical testability is discussed. Afterwards Neumann-Morgenstern utility theory is applied to the portfolio problem. Finally, it is shown how optimal risk allocation in an economy may lead to portfolio insurance.
\end{abstract}

\section{KEYWORDS}

Modern portfolio theory; Markowitz approach; capital asset-pricing model; Neumann-Morgenstern utilities; portfolio insurance.

\section{INTRODUCTION}

Starting with MARKOWITZ' (1952) pioneering work, modern portfolio theory has developed to a highly sophisticated field of research. In addition it became more and more obvious that for a large class of insurance problems a separate analysis of actuarial and financial risks is inappropriate. Of course modern portfolio theory is typically applied to common stocks. However, it can also be applied to bonds if there are risks with respect to default, exchange rates, inflation, etc. These facts, the increasing importance of new financial instruments, and the availability of computer capacities explain the growing interest of actuaries in modern portfolio theory.

In this paper some main results of modern portfolio theory are presented. However, some important aspects such as arbitrage pricing theory, multiperiod models, etc. are not treated here. ${ }^{1}$ The paper is organized as follows: Section 2 deals with the Markowitz approach. In Section 3 the Capital Asset Pricing Model (CAPM) is derived (SHARPE, 1963, 1964; LiNTNER, 1965; BLACK, 1972). Difficulties with respect to the testability of CAPM are discussed in Section 4 (ROLL, 1977). In Section 5 von Neumann-Morgenstern utility theory is applied to the portfolio problem and a generalized version of the CAPM-relationship is presented (CASS and STIGLITZ, 1970; MERTON, 1982). Finally, in Section 6 it is shown how the optimal risk allocation in an economy may lead to portfolio insurance (BORCH, 1960; LELAND, 1980).

1. For a rigorous and comprehensive representation of modern portfolio theory see INGERSOLL (1987). An intuitive introduction is given by COPELAND and WESTON (1988) or HARRINGTON (1987). 


\section{THE MARKOWITZ APPROACH}

Whereas in actuarial science the law of large numbers plays a central role this is not the case in portfolio theory. Due to the correlation between the returns on financial assets, diversification allows in general only for a reduction but not for an elimination of the risk. MARKOWITZ (1952) was the first who took the covariances between the rates of return into account.

\subsection{The Model}

There are $N$ assets $h=1, \ldots, N$ (e.g. common stocks). An investment of one unit of money (e.g. $1(\$)$ ) in asset $h$ leads to a stochastic return $R_{h}$. The first and second moments of $R_{1}, \ldots, R_{N}$ are assumed to exist.

The vector of expected values and the covariance matrix are denoted by

$$
\mu \in R^{N}, \quad \text { with } \quad \mu_{h}=E\left(R_{h}\right) \quad h=1, \ldots, N
$$

and

$$
V \in R^{N^{2}} \text {, with } V_{h l}=\operatorname{Cov}\left(R_{h}, R_{l}\right) \quad h, l=1, \ldots, N \text {. }
$$

A portfolio is given by a vector $\mathbf{x} \in R^{N}$, with $\sum_{h=1}^{N} x_{h}=1 . x_{h}$ denotes the weight of asset $h=1, \ldots, N .^{2}$ Hence the overall return on a portfolio $\mathrm{x}$ is given by the random variable

$$
R(\mathbf{x}):=\sum_{h=1}^{N} x_{h} R_{h}
$$

and one obtains immediately

$$
E[R(\mathbf{x})]=\boldsymbol{\mu}^{\prime} \mathbf{x}, \operatorname{Var}[R(\mathbf{x})]=\mathbf{x}^{\prime} V \mathbf{x} .
$$

DEFInITION. A portfolio $\mathbf{x}^{*}$ is called (mean-variance) efficient if there exists no portfolio $\mathbf{x}$ with

$$
E[R(\mathbf{x})] \geqslant E\left[R\left(\mathbf{x}^{*}\right)\right], \operatorname{Var}[R(\mathbf{x})]<\operatorname{Var}\left[R\left(\mathbf{x}^{*}\right)\right] .
$$

The Markowitz approach is a method to calculate mean-variance efficient portfolios. Hence, the Markowitz approach is based on mean-variance analysis, where the variance of the overall rate of return is taken as a risk measure and the expected value measures profitability. In contrast to expected utility maximization, mean-variance analysis takes into account only the first two moments and there is no clear theoretical foundation. The special assumptions under which mean-variance analysis is consistent with expected utility maximization are discussed in Section 5.

The Markowitz approach can be formalized as follows:

$$
\min _{\mathbf{x} \in R^{N}} 1 / 2 \mathbf{x}^{\prime} V \mathbf{x}
$$

2. $x_{h}<0$ corresponds to a short position with respect to asset $h$. 
subject to

$$
\begin{aligned}
& \mathbf{e}^{\prime} \mathbf{x}=1^{3} \\
& \boldsymbol{\mu}^{\prime} \mathbf{x} \geqslant r,
\end{aligned}
$$

where $r$ is the minimum level for the expected value of the overall rate of return. Here, the minimum level $r$ is assumed to be exogenously given. In applications, individual investors choose $r$ in accordance with their risk aversion.

$(M)$ is a quadratic convex optimization problem. Under the assumption

A.1 1) $V$ is positive definite,

2) e, $\mu$ are linearly independent,

there exists a unique solution of $(M)$ and the Kuhn-Tucker theorem can be applied. The Kuhn-Tucker conditions are given by

$$
\begin{aligned}
V \mathbf{x}-\lambda_{1} \mathbf{e}-\lambda_{2} \boldsymbol{\mu} & =0 \\
\mathbf{e}^{\prime} \mathbf{x} & =1 \\
\boldsymbol{\mu}^{\prime} \mathbf{x} & \geqslant r \\
\lambda_{2} \geqslant 0, \lambda_{2}\left(\boldsymbol{\mu}^{\prime} \mathbf{x}-r\right) & =0 .
\end{aligned}
$$

Hence

$$
\mathbf{x}=\lambda_{1} V^{-1} \mathbf{e}+\lambda_{2} V^{-1} \mu
$$

or if (2) is taken into account

$$
\mathbf{x}=\nu \mathbf{x}^{1}+(1-\nu) \mathbf{x}^{2}
$$

with

$$
\mathbf{x}^{1}=\frac{V^{-1} \mathbf{e}}{\mathbf{e}^{\prime} V^{-1} \mathbf{e}}, \quad \mathbf{x}^{2}= \begin{cases}\frac{V^{-1} \mu}{\mathbf{e}^{\prime} V^{-1} \mu} & \text { if } \mathbf{e}^{\prime} V^{-1} \mu \neq 0 \\ \mathbf{x}^{1}+V^{-1} \mu & \text { else }\end{cases}
$$

By varying the minimum level of return $r$, all mean-variance efficient portfolios are obtained. $x^{1}$ and $x^{2}$ do not depend on $r$. However. $\nu$ depends on $\lambda_{1}$ resp. on $\lambda_{2}$ and therefore ultimately on $r$. Hence, one gets

$$
\mathbf{x}(r)=\nu(r) \mathbf{x}^{1}+[1-\nu(r)] \mathbf{x}^{2} .
$$

Thus, any efficient portfolio is a combination of two fixed reference portfolios $x^{1}$ and $x^{2}$. Ingersoll $(1987$, p. 86$)$ shows that $x^{1}$ is the global minimum variance portfolio with an expected value of return

$$
r_{\min }=\frac{\mu^{\prime} V^{-1} \mathbf{e}}{\mathbf{e}^{\prime} V^{-1} \mathbf{e}}
$$

Furthermore, it is easily seen that (3) holds with equality if and only if $r \geqslant r_{\min }$. 3. $\mathbf{e}^{\prime}=(1,1, \ldots, 1) \in R^{N}$. 
Hence, for $r \geqslant r_{\min },(3)$ and (6) lead to

(8) $\sigma^{2}(r):=\operatorname{Var}\{R[\mathbf{x}(r)]\}=\nu^{2} \mathbf{x}^{\prime \prime} V \mathbf{x}^{1}+2 \nu(1-\nu) \mathbf{x}^{1} V \mathbf{x}^{2}+(1-\nu)^{2} \mathbf{x}^{\prime \prime} V \mathbf{x}^{2}$

(9) $\quad r=\nu \mu^{\prime} \mathbf{x}^{1}+(1-\nu) \mu^{\prime} \mathbf{x}^{2}$.

From (8) and (9) one can derive that there is a hyperbolic relationship between $r$ and $\sigma(r)$ (Figure 1).

\subsection{Availability of a riskless asset}

If, in addition to the risky asset $h=1, \ldots, N$ (common stocks, etc.), a riskless asset $h=0$ with a deterministic return $R_{0}$ (e.g. a treasury bill) is available, the model changes as follows:

$$
\begin{aligned}
& \tilde{\mu}=\left(\mu_{0}, \mu\right) \in R^{N+1}, \quad \text { with } \quad \mu_{0}=R_{0}, \mu_{h}=E\left(R_{h}\right), \quad h=1, \ldots, N \\
& V \in R^{N^{2}}, \quad \text { with } \quad V_{h l}=\operatorname{Cov}\left(R_{h}, R_{l}\right), \quad h, l=1, \ldots, N \\
& \tilde{\mathbf{x}}=\left(x_{0}, \mathbf{x}\right) \in R^{N+1}, \quad \text { with } \quad \sum_{h=0}^{N} x_{h}=1
\end{aligned}
$$

$R(\tilde{\mathbf{x}}):=\sum_{h=0}^{N} x_{h} R_{h}$

Mean-variance efficient portfolios now result from the optimization problem

$$
\min _{\tilde{\mathbf{x}} \in R^{N+1}} 1 / 2 \mathbf{x}^{\prime} V \mathbf{x}
$$

subject to

$\left(M^{\prime}\right)$

$$
\begin{gathered}
\tilde{\mathbf{e}}^{\prime} \tilde{\mathbf{x}}=1^{4} \\
\tilde{\boldsymbol{\mu}}^{\prime} \tilde{\mathbf{x}} \geqslant r .
\end{gathered}
$$

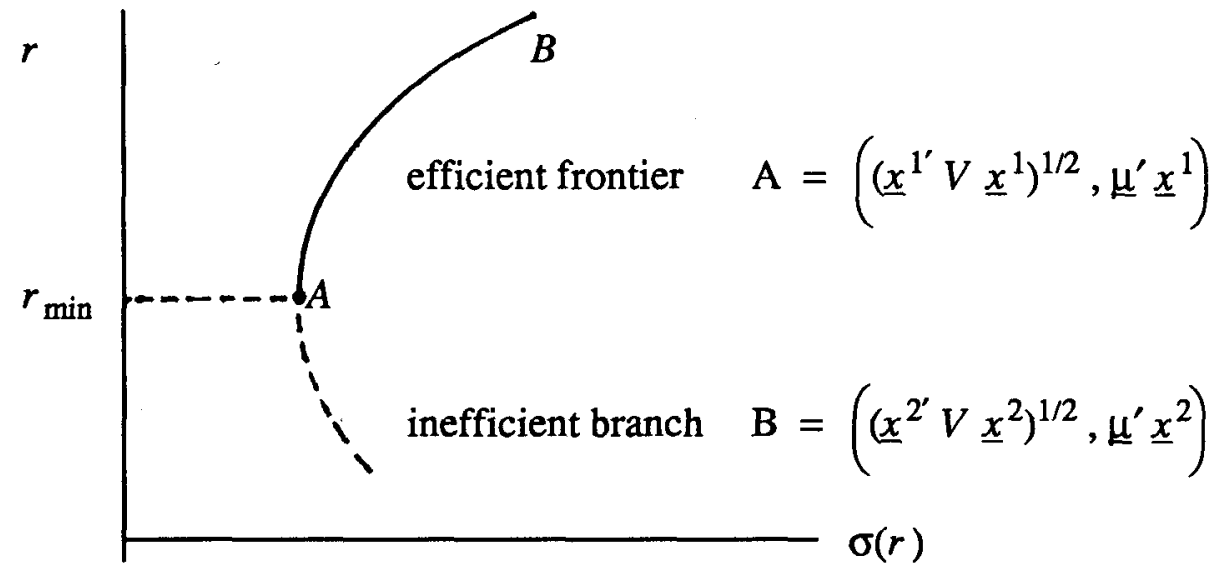

Figure 1.

4. $\tilde{\mathbf{e}}^{\prime}=(1,1, \ldots, 1) \in R^{N+1}$. 
Under the assumption

$\mathrm{A}^{\prime} 1$ 1) $V$ is positive definite

2) $\tilde{\mathbf{e}}, \tilde{\boldsymbol{\mu}}$ are linearly independent

mean-variance efficient portfolios are still of the form

$$
\tilde{\mathbf{x}}(r)=\nu(r) \tilde{\mathbf{x}}^{1}+[1-\nu(r)] \tilde{\mathbf{x}}^{2}
$$

but now one can show that

$$
\tilde{\mathbf{x}}^{1^{\prime}}=(1,0, \ldots, 0), \tilde{\mathbf{x}}^{2^{\prime}}=\left(0, x_{1}^{2}, \ldots, x_{N}^{2}\right)
$$

holds, i.e. every mean-variance efficient portfolio is a combination of the riskless investment $\tilde{\mathbf{x}}^{\mathbf{l}}$ with a reference portfolio $\tilde{\mathbf{x}}^{2}$, consisting exclusively of risky assets.

Furthermore, the efficient frontier degenerates to a straight line (Figure 2).

\subsection{Remarks}

1) It is important to note that the special structure of the set of mean-variance efficient portfolios provides the basis for the Capital Asset Pricing Model (see Section 3).

2) In practical applications additional constraints sometimes have to be imposed, such as exclusion of short sales, bounds on the weights of individual assets, etc. With constraints of this type, the optimization problem is still quadratic convex and powerful numerical methods are available. However, in general the special structure of the efficient frontier is destroyed.

3) A detailed and careful analysis of the Markowitz approach can be found in the appendix of ROLL's (1977) article or in INGERSOLL's (1987) chapter 4.

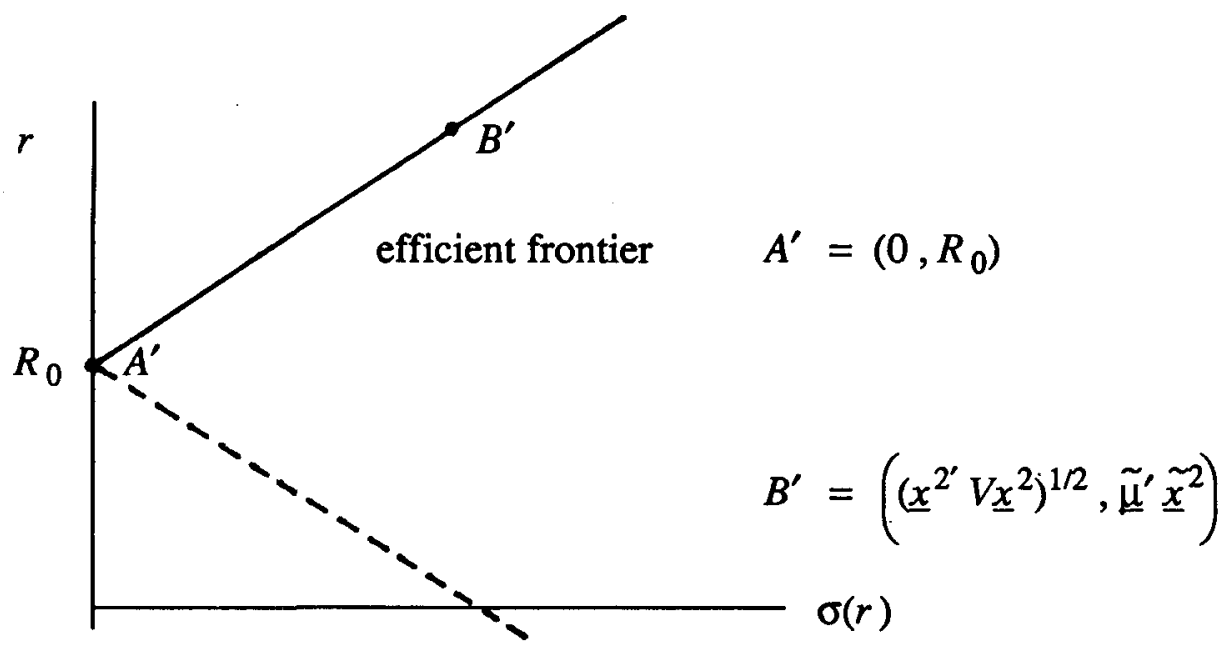

FIGURE 2 . 
3. THE CAPITAL ASSET PRICING MODEL (CAPM)

\subsection{The Sharpe-Lintner Model}

In the Sharpe-Lintner Model (SHARPE, 1963, 1964; LINTNER, 1965), there is a riskless asset $h=0$ and $N$ risky assets $h=1, \ldots, N$.

There are $m$ investors $i=1, \ldots, m$ who are characterized as follows:

1) $W_{i}>0$ is the initial wealth of investor $i$.

2) Investors $i=1, \ldots, m$ agree on the first and second moments of returns on assets $h=0, \ldots, N$, i.e.

$$
\tilde{\boldsymbol{\mu}}^{i}=\tilde{\boldsymbol{\mu}}, \quad V^{i}=V \quad i=1, \ldots, m,
$$

3) Investor $i$ seeks a mean-variance efficient portfolio $\tilde{\mathbf{x}}_{i}$ with $E\left[R\left(\tilde{\mathbf{x}}_{i}\right)\right]=r_{i}$, where $r_{i}>R_{0} i=1, \ldots, m$.

According to formula (6 $6^{\prime}$ ) (Section 2.2) the portfolio chosen by investor $i$ is of the form

$$
\tilde{\mathbf{x}}_{i}=\nu\left(r_{i}\right) \tilde{\mathbf{x}}^{1}+\left[1-\nu\left(r_{i}\right)\right] \tilde{\mathbf{x}}^{2} \quad i=1, \ldots, m .
$$

Total demand results in a portfolio

$$
\tilde{\mathbf{x}}_{d}^{M}:=\frac{1}{W} \sum_{i=1}^{m} W_{i} \tilde{\mathbf{x}}_{i}, \quad \text { with } \quad W:=\sum_{i=1}^{m} W_{i} .
$$

By means of $\left(6^{\prime}\right)$ one can show (See Fig. 3) that $\tilde{\mathbf{x}}_{d}^{M}$ lies on the efficient frontier and can be represented as the solution of

subject to

$$
\min _{\tilde{\mathbf{x}} \in R^{N+1}} 1 / 2 \mathbf{x}^{\prime} V \mathbf{x}
$$

$\left(M^{\prime \prime}\right)$

$$
\begin{gathered}
\tilde{\mathbf{e}}^{\prime} \tilde{\mathbf{x}}=1 \\
\tilde{\boldsymbol{\mu}}^{\prime} \tilde{\mathbf{x}} \geqslant r_{M}
\end{gathered}
$$

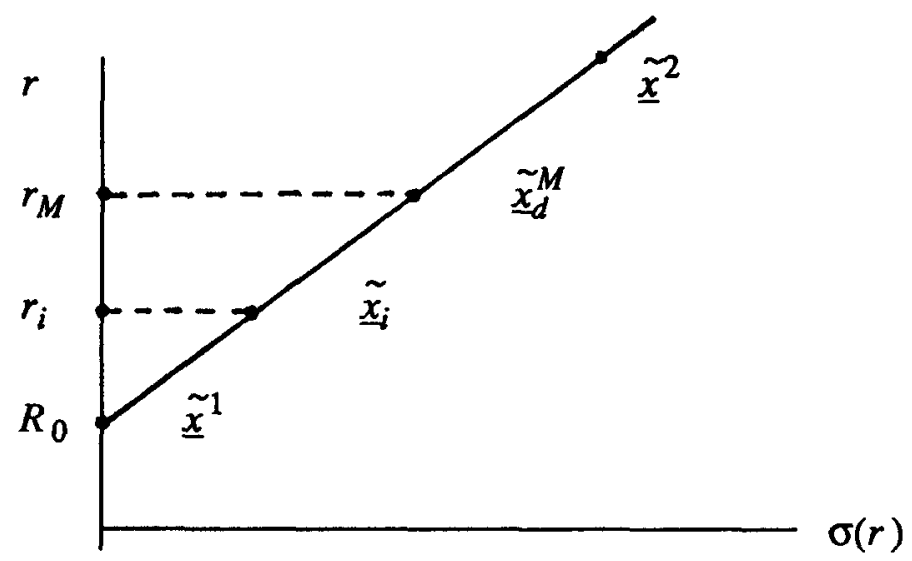

Figure 3 . 
with

$$
r_{M}:=\frac{1}{W} \sum_{i=1}^{m} W_{i} r_{i}
$$

Hence, $\tilde{\mathbf{x}}_{d}^{M}=\left(\mathrm{x}_{d 0}^{M}, \mathbf{x}_{d}^{M}\right)$ satisfies the Kuhn-Tucker conditions

$$
\begin{aligned}
-\lambda_{1}-\lambda_{2} R_{0} & =0 \\
V \mathbf{x}_{d}^{M}-\lambda_{1}-\lambda_{2} \mu & =0 \\
\tilde{\mathbf{e}}^{\prime} \tilde{\mathbf{x}}_{d}^{M} & =1 \\
\tilde{\mu}^{\prime} \tilde{\mathbf{x}}_{d}^{M} & =r_{M} \\
\lambda_{2} & \geqslant 0 .
\end{aligned}
$$

Formulae (11) and (12) lead to

$$
V \mathbf{x}_{d}^{M}=\lambda_{2}\left(\mu-R_{0} \mathbf{e}\right) .
$$

From (11), (12) and $\mu_{0}=R_{0}$ one obtains

$$
\mathbf{x}_{d}^{M^{\prime}} V \mathbf{x}_{d}^{M}=\lambda_{1} \tilde{\mathbf{e}}^{\prime} \tilde{\mathbf{x}}_{d}^{M}+\lambda_{2} \tilde{\mu}^{\prime} \tilde{\mathbf{x}}_{d}^{M}
$$

or according to (11), (13) and (14)

$$
\mathbf{x}_{d}^{M^{\prime}} V \mathbf{x}_{d}^{M}=\lambda_{2}\left(r_{M}-R_{0}\right) .
$$

Formulac (16) and (18) imply

$$
\frac{V \mathbf{x}_{d}^{M}}{\mathbf{x}_{d}^{M^{\prime}} V \mathbf{x}_{d}^{M}}=\frac{\mu-R_{0} \mathbf{e}}{r_{M}-R_{0}}
$$

On the other hand, total supply is given by the market portfolio $\tilde{\mathbf{x}}^{M}$, where all assets are held in proportion to their market values. In equilibrium total demand must be equal to total supply, i.e.

$$
\tilde{\mathbf{x}}_{d}^{M}=\tilde{\mathbf{x}}^{M} .
$$

Combining (19) and (20) leads to the equilibrium condition

$$
\frac{V \mathbf{x}^{M}}{\mathbf{x}^{M^{\prime}} V \mathbf{x}^{M}}=\frac{\mu-R_{0} \mathbf{e}}{r_{M}-R_{0}}
$$

or by taking into account

$$
\begin{aligned}
\mu_{h} & =E\left(R_{h}\right), \quad h=1, \ldots, N \\
r_{M} & =E\left(R_{M}\right), \quad \text { where } \quad R_{M}=\sum_{h=0}^{N} x_{h}^{M} R_{h} \\
\mathbf{x}^{M^{\prime} V \mathbf{x}^{M}} & =\operatorname{Var}\left(R_{M}\right) \\
\mathbf{x}^{M^{\prime}} V & =\left(\operatorname{Cov}\left(R_{1}, R_{M}\right), \ldots, \operatorname{Cov}\left(R_{N}, R_{M}\right)\right)
\end{aligned}
$$

one obtains the CAPM-relationship

$$
E\left(R_{h}\right)-R_{0}=\frac{\operatorname{Cov}\left(R_{h}, R_{M}\right)}{\operatorname{Var}\left(R_{M}\right)}\left[E\left(R_{M}\right)-R_{0}\right], \quad h=1, \ldots, N
$$




\section{COMMENTS}

1) $\beta_{h}:=\operatorname{Cov}\left(R_{h}, R_{M}\right) / \operatorname{Var}\left(R_{M}\right)$ is called the beta-coefficient of asset $h$.

$E\left(R_{h}\right)-R_{0}$ is the risk premium on asset $h$

$E\left(R_{M}\right)-R_{0}$ is the risk premium on the market portfolio.

2) Under stationarity and normality assumptions the beta-coefficients can be estimated by applying the ordinary least square method to

$$
R_{h}-R_{0}=\alpha_{h}+\beta_{h}\left(R_{M}-R_{0}\right)+\varepsilon_{h} .
$$

The term $\beta_{h}\left(R_{M}-R_{0}\right)$ corresponds to the systematic risk which is undiversifiable. The error term $\varepsilon_{h}$ satisfies $\operatorname{Cov}\left(\varepsilon_{h}, R_{M}\right)=0$ and corresponds to the unsystematic risk. In particular, the following decomposition is possible

$$
\operatorname{Var}\left(R_{h}\right)=\beta_{h}^{2} \operatorname{Var}\left(R_{M}\right)+\operatorname{Var}\left(\varepsilon_{h}\right)
$$

3) For empirical beta estimation there is no general agreement with respect to the measurement period and the interval choice. Monthly data over a five-year period are widely used.

4) Typically the observed beta-coefficients are positive. However, negative betacoefficients may occur as well. ${ }^{5}$

5) According to the CAPM relationship each asset $h$ can be represented by a point $\left(\beta_{h}, E\left(R_{h}\right)\right)$ which lies on the theoretical market line

$$
E\left(R_{h}\right)=R_{0}+\left[E\left(R_{M}\right)-R_{0}\right] \beta_{h}
$$

Often the empirical market line which is based on estimations for $\beta_{h}, E\left(R_{h}\right)$, $E\left(R_{M}\right)$ deviates substantially (see, e.g. BLACK, JENSEN and SCHOLES, 1972).

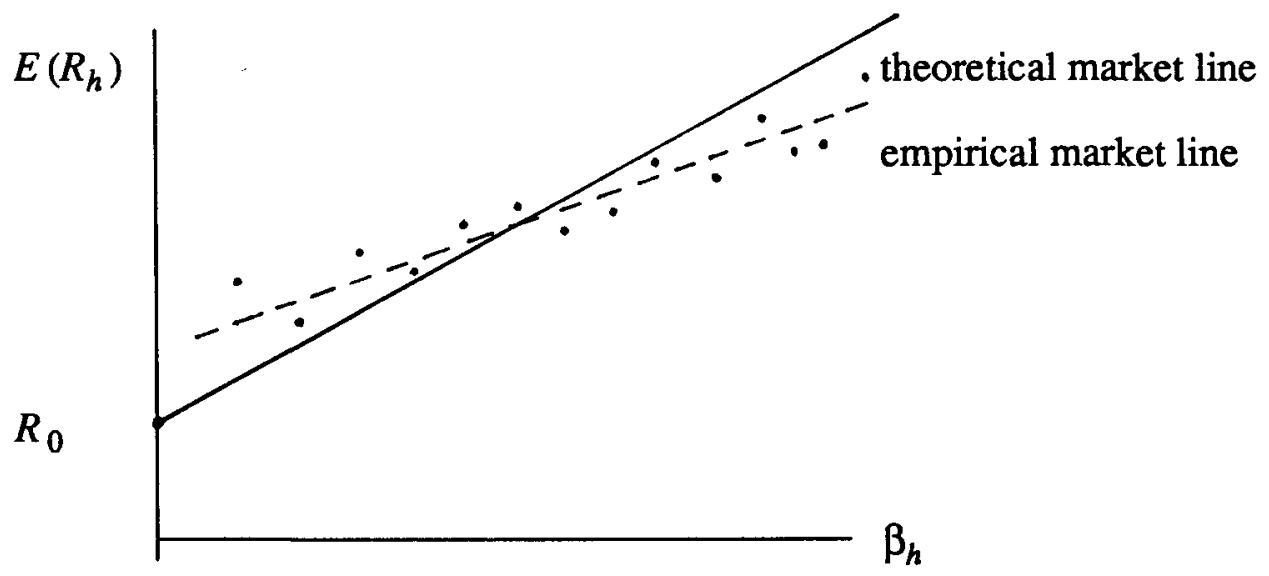

FIGURE 4.

5. In such a case $R_{h}$ and $R_{M}$ are negatively correlated and $E\left(R_{h}\right)<R_{0}$ must hold. 


\subsection{The Black Model}

To assume the existence of a riskless asset is somewhat questionable, especially if one is interested in real returns. Fortunately, even without assuming the existence of a riskless asset a CAPM-relationship can be derived.

It is assumed that the framework of Section 2.1 holds and that there are $m$ investors $i=1, \ldots, m$ with

initial wealth $W_{i}$,

first and second moments of returns given by $\mu^{i}=\mu, V^{i}=V$

and seeking for mean-variance efficient portfolios $\mathbf{x}_{i}$ with $E\left[R\left(\mathbf{x}_{i}\right)\right]=r_{i}$, where $r_{i} \geqslant r_{\min }, i=1, \ldots, m$.

Under these conditions it can easily be shown that in equilibrium the market portfolio $\mathbf{x}^{M}$ must be efficient and from the corresponding optimality conditions one can derive the CAPM-relationship

$$
\begin{aligned}
E\left(R_{h}\right)-E\left[R\left(\mathbf{x}^{0}\right)\right] & =\frac{\operatorname{Cov}\left(R_{h}, R_{M}\right)}{\operatorname{Var}\left(R_{M}\right)}\left\{E\left(R_{M}\right)-E\left[R\left(\mathbf{x}^{0}\right]\right\}\right. \\
h & =1, \ldots, N .
\end{aligned}
$$

$\mathbf{x}^{0}$ is the so-called zero-beta portfolio with the properties

a) $\operatorname{Cov}\left(R_{M}, R\left(\mathbf{x}^{0}\right)\right)=0$.

b) There exists $\nu$ such that (5) holds, i.e. $\mathbf{x}^{0}=\nu \mathbf{x}^{1}+(1-\nu) \mathbf{x}^{2}$.

\section{EXPLANATIONS}

1) According to $b$ ) the point $Z:=\left(\left(\mathbf{x}^{0^{\prime}} V \mathbf{x}^{0}\right)^{1 / 2}, \mu^{\prime} \mathbf{x}^{0}\right)$ lies on the hyperbola described in Section 2.1.

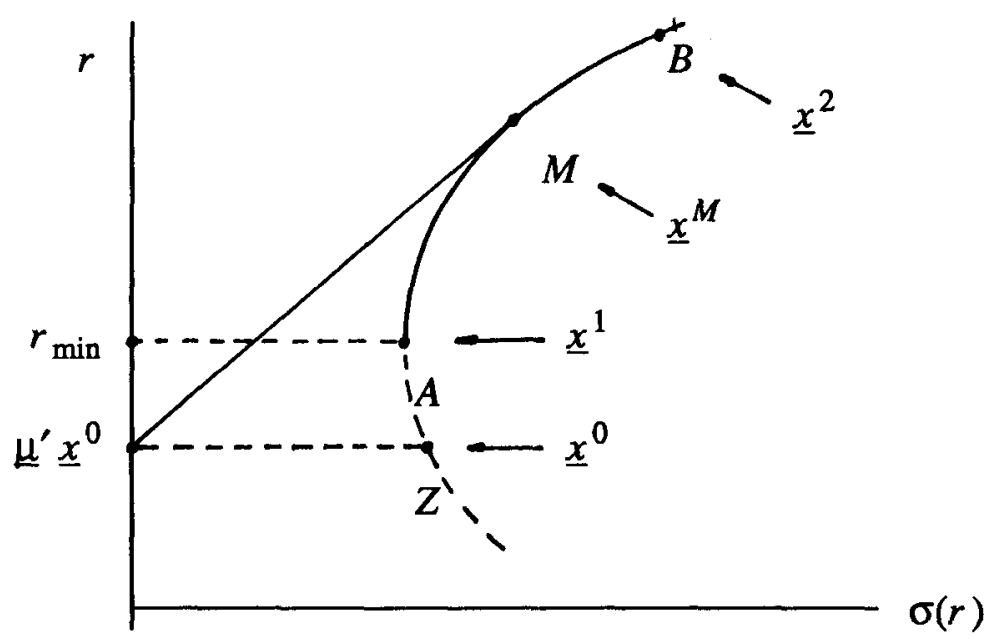

Figure 5. 
2) ROLL (1977) shows that:

$Z$ lies on the inefficient branch of $H$.

The tangent in the point $M=\left(\left(\mathbf{x}^{M^{\prime}} V \mathbf{x}^{M}\right)^{1 / 2}, \mu^{\prime} \mathbf{x}^{M}\right)$ intersects the return axis at $\left(0, \mu^{\prime} \mathbf{x}^{0}\right)$.

COMMENTS. This model was developed by BLACK (1972). It is more than an interesting alternative to the Sharpe-Lintner approach. In the next section we shall see that it is needed in order to discuss the empirical testability of the Sharpe-Lintner model.

\section{EMPIRICAL TESTABILITY OF THE SHARPE-LINTNER MODEL}

Before discussing testability we must recall that the Sharpe-Lintner model was developed under restrictive assumptions, namely:

M.1 Evaluation of portfolios by mean-variance analysis,

M.2 Uniform planning horizon for all investors,

M.3 Homogeneous expectations,

M.4 Existence of a riskless asset,

M.5 Exclusion of transaction costs,

M.6 No restrictions on short sales.

BlACK et al. (1972), BLUME and Friend (1973), FAMA and MACBETH (1973) were among the first to test the Sharpe-Lintner model. The evidence provided by their studies in favour of the CAPM-relationship is rather weak. ${ }^{6}$ However, as ROLL (1977) pointed out there is a serious problem with the empirical testability of the Sharpe-Lintner model. Due to the fact that all types of bonds, real estate, etc. should be contained in the market portfolio, this portfolio cannot be reasonably approximated.

In order to analyse the consequences of this fact, the following points of Roll's paper are particularly important:

P.1 Within the framework of the Black model the CAPM-relationship (23) holds for any mean-variance efficient portfolio $x^{q} .{ }^{7}$ On the other hand, if there is a linear relationship

$$
E\left(R_{h}\right)=a \beta_{h}+b,
$$

with

$$
\beta_{h}:=\frac{\operatorname{Cov}\left(R_{h}, R\left[\mathbf{x}^{q}\right]\right)}{\operatorname{Var}\left(R\left[\mathbf{x}^{q}\right]\right)}, \quad h=1, \ldots, N
$$

the portfolio $x^{q}$ is mean-variance efficient (loc, cit., corollary 6 , pp. 165-166).

P.2 There is an interesting connection between the Black and the

6. In fact BLACK et al. (1972) and BLUME and FRIEND (1973) reject the Sharpe-Lintner model.

7. $x^{0}$ is the corresponding zero-beta portfolio. 
Sharpe-Lintner models. To see this, let $h=0$ be the riskless and $h=1, \ldots, N$ the risky assets. Then the efficient frontier is a straight line $L$ (see Section 2.2). If attention is restricted to portfolios consisting only of risky assets a constrained efficient frontier $C$ results which is the upper branch of a hyperbola (see Section 2.1). Obviously $L$ must be tangential to $C$ (Figure 6).

From now on, it is assumed that the riskless asset $h=0$ is in zero net supply, i.e. $x_{0}^{M}=0 .{ }^{8}$ Then, in equilibrium the market portfolio $\mathrm{x}^{M}$ must correspond to the tangency point $T$ (Figure 6). Hence, in equilibrium $\mathbf{x}^{M}$ satisfies the CAPMrelationship for the Black model

$$
E\left(R_{h}\right)-E\left[R\left(\mathbf{x}^{0}\right)\right]=\frac{\operatorname{Cov}\left(R_{h}, R\left[\mathbf{x}^{M}\right]\right)}{\operatorname{Var}\left(R\left[\mathbf{x}^{M}\right]\right)}\left\{E\left(R\left[\mathbf{x}^{M}\right]\right)-E\left(R\left[\mathbf{x}^{0}\right]\right)\right\}
$$

However, due to explanation 2 (Section 3.2)

$$
E\left(R\left[\mathbf{x}^{0}\right]\right)=R_{0}
$$

holds and one obtains the CAPM-relationship for the Sharpe-Lintner model

$$
E\left(R_{h}\right)-R_{0}=\frac{\operatorname{Cov}\left(R_{h}, R\left[\mathbf{x}^{M}\right]\right)}{\operatorname{Var}\left(R\left[\mathbf{x}^{M}\right]\right)}\left\{E\left(R\left[\mathbf{x}^{M}\right]\right)-R_{0}\right\}, h=1, \ldots, N .
$$

P.3 Suppose that the Sharpe-Lintner model is true, i.e. the market portfolio $\mathbf{x}^{M}$ satisfies the CAPM-relationship (22). If the market proxy $\mathbf{x}^{p}$ is different from $\mathbf{x}^{M}$ two possibilities arise.

1) $\mathbf{x}^{p}=\mathbf{x}^{m^{\prime}}$, where $\mathbf{x}^{m^{\prime}}$ does not belong to the constrained efficient frontier $C$ (Fig. 7). Due to P.1 the relationship (24) is no longer satisfied and the application of statistical methods leads in general to a rejection of the Sharpe-Lintner model.

2) $\mathbf{x}^{p}=\mathbf{x}^{m}$, where $\mathbf{x}^{m}$ belongs to the constrained efficient frontier $C$ (Figure

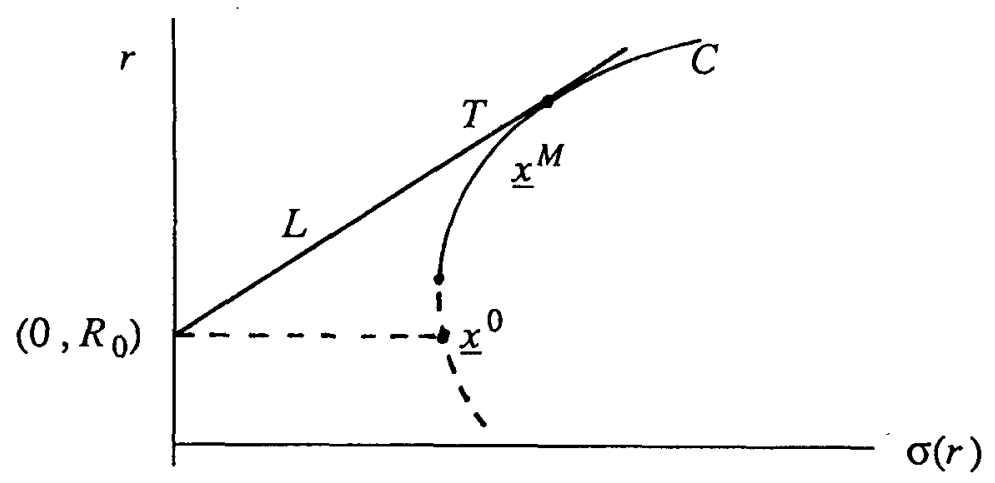

FIGURE 6.

8. This condition can be satisfied by choosing an appropriate normalization. 


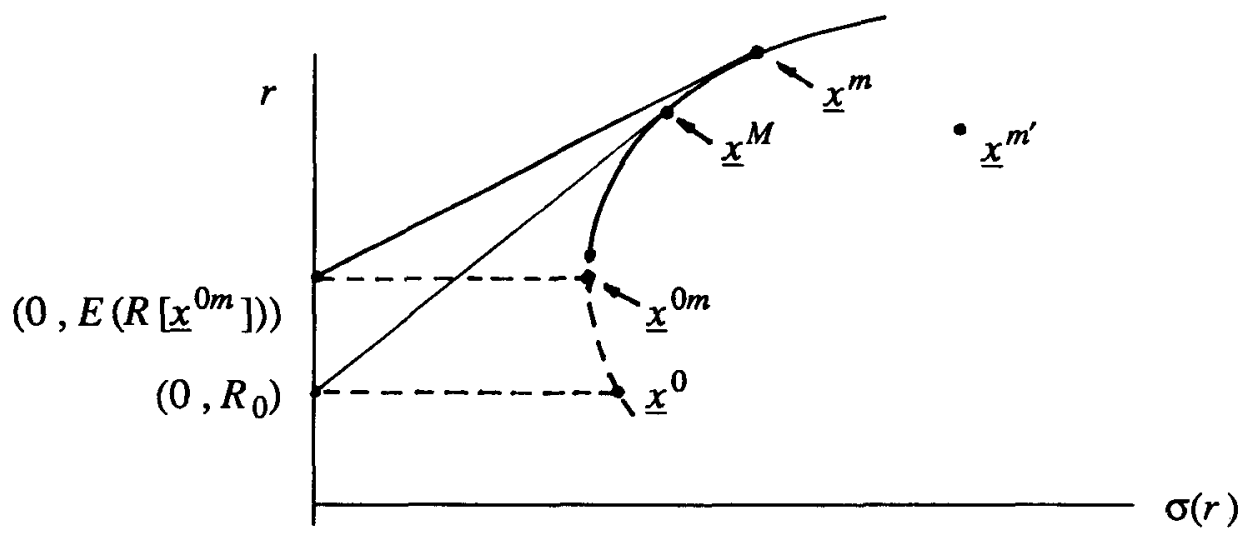

FIGURE 7.

7). Then, according to P.1,

$$
\begin{aligned}
& E\left(R_{h}\right)-E\left(R\left[\mathbf{x}^{0 m}\right]\right)=\frac{\operatorname{Cov}\left(R_{h}, R\left[\mathbf{x}^{m}\right]\right)}{\operatorname{Var}\left(R\left[\mathbf{x}^{m}\right]\right)}\left\{E\left(R\left[\mathbf{x}^{m}\right]\right)-E\left(R\left[\mathbf{x}^{0 m}\right]\right)\right\} \\
& \text { holds. }^{9} \text { However, } \mathbf{x}^{m} \neq \mathbf{x}^{M} \text { (Figure 7) leads to } h=1, \ldots, N
\end{aligned}
$$

$$
E\left(R\left[\mathbf{x}^{0 m}\right]\right) \neq R_{0}
$$

and again the application of statistical methods leads in general to a rejection of the Sharpe-Lintner model.

Roll's critique gave rise to consternation among researchers. In a recent paper Shanken (1987) presented a method to test the joint hypothesis that the Sharpe-Lintner model is true and that the correlation coefficient $\rho\left(R\left[\mathbf{x}^{M}\right], R\left[\mathrm{x}^{m}\right]\right)$ between the returns of the market portfolio $\mathrm{x}^{M}$ and the proxy $\mathbf{x}^{m}$ exceeds a given limit $\bar{\rho}$. For the equal-weighted CRSP index (an American stock index developed by the Center of Research in Security Prices at the University of Chicago) and a limit for the correlation coefficient of $\bar{\rho}=0.7$ he had to reject the joint hypothesis at the $95 \%$ confidence level. With multivariate proxies consisting of a stock and a bond index Shanken reached similar conclusions.

\section{APPLICATION OF VON NEUMANN-MORGENSTERN UTILITY THEORY}

Mean-variance analysis is rather unsatisfactory on theoretical grounds. Fortunately, NM utility theory can be applied to the portfolio problem.

Throughout this section it is assumed that there is a riskless asset $h=0$ with a deterministic return $R_{0}$ and $N$ risky assets $h=1, \ldots, N$ with stochastic returns $R_{h}$. The overall return $R(\tilde{\mathbf{x}})$ of a portfolio $\tilde{\mathbf{x}}=\left(x_{0}, x_{1}, \ldots, x_{N}\right)$ is evaluated by a NM utility function $u: R \rightarrow R$.

9. $\mathbf{x}^{0 m}$ is the zero-beta portfolio which corresponds to $x^{\prime \prime}$. 


\subsection{Efficient Portfolios}

DEFinition 1. A portfolio $\tilde{\mathbf{x}}^{*}$ is called optimal relative to the NM utility $u: R \rightarrow R$ if it is a solution of the optimization problem

$$
\max _{\tilde{\mathbf{x}} \in R^{N+1}} E\{u[R(\tilde{\mathbf{x}})]\}^{10,11}
$$

subject to

$$
\sum_{h=0}^{N} x_{h}=1
$$

REMARK. The optimization problem $(0)$ is equivalent to

$$
\max _{\left(x_{1}, \ldots, x_{N}\right) \in R^{N}} E\left\{u\left[R_{0}+\sum_{h=1}^{N} x_{h}\left(R_{h}-R_{0}\right)\right]\right\} .
$$

\section{ASSUMPTIONS}

B.1 The NM utility $u: R \rightarrow R$ is increasing, strictly concave and continuously differentiable.

B.2 The random variables $R_{h}, h=1, \ldots, N$ are bounded.

Proposition. Under B. 1 and B.2 the portfolio $\tilde{\mathbf{x}}^{*}$ is optimal relative to $u$ if and only if

$$
E\left\{u^{\prime}\left[R\left(\tilde{\mathbf{x}}^{*}\right)\right]\left(R_{h}-R_{0}\right)\right\}=0, h=1, \ldots, N
$$

PROOF.

1) Under B.1 and B.2 the objective function of $\left(0^{\prime}\right)$ is well defined and concave in $\left(x_{1}, \ldots, x_{N}\right)$.

2) Due to B.1 and B.2 Lebesgue's theorem allows to reverse the order of differentiation and integration. ${ }^{12}$

DEFinition 2. A portfolio $\tilde{\mathbf{x}}^{*}$ is called efficient if there exists a NM utility $v: R \rightarrow R$ satisfying B.1 such that $\tilde{\mathbf{x}}^{*}$ is optimal relative to $v$.

\subsection{Mutual Fund Theorems}

In Section 2 it was shown that the set of mean-variance efficient portfolios can always be spanned by two reference portfolios. In the framework of NM utility theory this is no longer the case. ${ }^{13}$ However, if there are restrictions on the class

10. $R(\tilde{\mathbf{x}}):=\sum_{h=0}^{N} x_{h} R_{h}$.

11. If $W_{0}$ is the initial wealth, then of course one has to evaluate the final wealth $W_{0} R(\tilde{\mathbf{x}})$. However, the choice of an appropriate scaling allows always for the normalization $W_{0}=1$.

12. This problem is of ten overlooked in the literature.

13. Since NM utility theory takes third and higher moments of the overall return on a portfolio into account one can show that the set of efficient portfolios becomes larger than under mean-variance analysis. 
of NM utilities and/or the class of returns distributions, the set of efficient portfolios can still be spanned by a few references portfolios.

RESTRICTIONS ON RETURNS DISTRIBUTIONS. The following result is often presented as a theoretical basis for mean-variance analysis in the case of multivariate normal distribution of returns.

THEOREM. Suppose that $\mathbf{R}=\left(R_{1}, \ldots, R_{N}\right)$ has a multivariate normal probability distribution with $a$ density $f(\mathbf{r})=(2 \pi)^{-N / 2}(\operatorname{det} \quad V)^{-1 / 2}$ $\exp \left\{-1 / 2(\mathbf{r}-\boldsymbol{\mu})^{\prime} \boldsymbol{V}^{-1}(\mathbf{r}-\boldsymbol{\mu})\right\}$, where $V$ is a regular $N \times N$ covariance matrix.

Then, the set of efficient portfolios is spanned by two reference portfolios $\tilde{\mathbf{x}}^{(1)}, \tilde{\mathbf{x}}^{(2)}$, where

$\tilde{\mathbf{x}}^{(1)}=(1,0, \ldots, 0) \in R^{N+1}$ is the riskless investment

$\tilde{\mathbf{x}}^{(2)}$ is a fixed mean-variance efficient portfolio.

ProOf. See MERTON (1982, theorem 4.11, p. 631) or ROSS (1978, pp. 272-273).

RESTRICTIONS ON THE CLASS OF NM UTILITIES. Let $U$ be a class of NM utilities $u: R \rightarrow R$. The set of portfolios which are optimal relative to some $u \in U$ is denoted by $\Psi^{e}(U)$. HAKANSSON (1969) and CASS and STIGLITZ (1970) were the first to look for classes $U$ such that $\Psi^{e}(U)$ is spanned by two reference portfolios $\tilde{\mathbf{x}}^{(1)}$ and $\tilde{\mathbf{x}}^{(2)}$, where $\tilde{\mathbf{x}}^{(1)}=(1,0, \ldots, 0)$. Under regularity assumptions ${ }^{14}$ with respect to returns distribution they show that the following classes $U(c)$, $c \in(-\infty, 0) \cup(0, \infty]$ have this property:
a) $U(c)=\left\{u \mid u(w)=\frac{\left(w-\hat{W}_{k}\right)^{1-c}}{1-c}, w \geqslant \hat{W}_{k}\right\} \forall c \in(0,1) \cup(1, \infty)$
b) $U(c)=\left\{u \mid u(w)=-\frac{\left(\hat{W}_{k}-w\right)^{1-c}}{1-c}, w \leqslant \hat{W}_{k}\right\} \quad \forall c \in(-\infty, 0)$
c) $U(c)=\left\{u \mid u(w)=\ln \left(w-\hat{W}_{k}\right), w \geqslant \hat{W}_{k}\right\}, \quad c=1$
d) $U(c)=\left\{u \mid u(w)=-\exp \left(-\delta_{k} w\right)\right\}, \quad c=\infty$

where $\hat{W}_{k}$ can be interpreted as the subsistence level of wealth in a) and c) and as the satiation level of wealth in b) (see INGERSOLL, 1987, pp. 146-147).

\section{COMMENTS}

1) These classes are also important in risk theory. According to Borch's theorem there is linear risk sharing within each class.

2) The union of these classes represents the HARA-class (Hyperbolic Absolute

14. A full specification of these conditions is beyond the scope of this paper. 
Risk Aversion), which is characterized by

$$
-\frac{u^{\prime \prime}(w)}{u^{\prime}(w)}=\frac{1}{a+b w}>0
$$

3) For $c=-1$ the class of quadratic NM utilities results and mean-variance analysis is obtained as a special case.

\subsection{A risk Measure for Individual Securities}

In mean-variance analysis the beta-coefficients are used to measure the risk of an individual security relative to the market portfolio. The NM utility framework allows for the following generalization:

DEFINITION 3. Let $\tilde{\mathbf{x}}^{K}$ be an optimal portfolio relative to a NM utility $u: R \rightarrow R$ satisfying B.1. Then

$$
b_{h}^{K}:=\frac{\operatorname{Cov}\left(u^{\prime}\left[R\left(\tilde{\mathbf{x}}^{K}\right)\right], R_{h}\right)}{\operatorname{Cov}\left(u^{\prime}\left[R\left(\tilde{\mathbf{x}}^{K}\right)\right], R\left(\tilde{\mathbf{x}}^{K}\right)\right)} \quad h=1, \ldots, N
$$

is called the measure of risk of asset $h$ relative to portfolio $\tilde{\mathbf{x}}^{K}$.

REMARK. $b_{h}^{K}$ coincides with the beta-coefficient of asset $h$ if $\tilde{\mathbf{x}}^{K}$ is the market portfolio and if

$u$ is quadratic

or

$u$ is twice differentiable and $\mathbf{R}=\left(R_{\mathrm{l}}, \ldots, R_{N}\right)$ has a multivariate normal distribution (see INGERSOLL, 1987, pp. 13-14).

PROPERTIES OF $b_{h}^{K}$.

P.1 If $\tilde{\mathbf{x}}^{k}$ is an optimal portfolio relative to a NM utility $u$ satisfying B. 1 and if some regularity conditions are satisfied then

$$
E\left(R_{h}\right)-R_{0}=b_{h}^{K}\left\{E\left[R\left(\tilde{\mathbf{x}}^{K}\right)\right]-R_{0}\right\}, \quad h=1, \ldots, N
$$

holds.

P.2 Under regularity conditions, for all efficient portfolios $\tilde{\mathbf{x}}^{K}, \tilde{\mathbf{x}}^{L}$

$$
\begin{gathered}
b_{h}^{K}=b_{h^{\prime}}^{K} \Leftrightarrow b_{h}^{L}=b_{h^{\prime}}^{L} \\
b_{h}^{K}>b_{h^{\prime}}^{K} \Leftrightarrow b_{h}^{L}>b_{h^{\prime}}^{L}
\end{gathered} \quad h, h^{\prime} \in\{1, \ldots, N\}
$$

holds.

For a proof of P.1 and P.2 see INGERSOLL (1987, p. 134).

INTERPRETATION OF P.1 AND P.2.

ad P.1 If there are $m$ investors with homogeneous expectations, whose 
preferences can be represented by NM utilities, $u^{i}, i=1, \ldots, m$, then in general the market portfolio is not efficient (see INGERSOLL, 1987, pp. 140-143). However, in the special case, where

$$
u^{i} \in U(c), i=1, \ldots, m \text {, for some } c \in(-\infty, 0) \cap(0, \infty], \text { [see (27)] , }
$$

there exists $u_{M} \in U(c)$ such that the market portfolio $\tilde{\mathbf{x}}^{M}$ is optimal relative to $u_{M}$. Then, P.1 leads to the following generalized version of the CAPM-relationship

$$
E\left(R_{h}\right)-R_{0}=\frac{\operatorname{Cov}\left(u_{M}^{\prime}\left[R\left(\tilde{\mathbf{x}}^{M}\right)\right], R_{h}\right)}{\operatorname{Cov}\left(u_{M}^{\prime}\left[R\left(\tilde{\mathbf{x}}^{M}\right)\right], R\left(\tilde{\mathbf{x}}^{M}\right)\right)}\left\{E\left[R\left(\tilde{\mathbf{x}}^{M}\right)\right]-R_{0}\right\},
$$

$$
h=1, \ldots, N \text {. }
$$

ad P.2 $b_{h}^{K}$ leads to a complete pre-ordering on the set of securities $\{1, \ldots, N\}$. According to P.2, this pre-ordering does not depend on the underlying efficient portfolio $\tilde{\mathbf{x}}^{K}$.

\section{PORTFOLIO INSURANCE}

Throughout this section, it is assumed that there is a riskless asset with a deterministic return $R_{0}$ and a reference portfolio with the stochastic return $R_{M}$. Usually, the protection of the reference portfolio by a put option is called portfolio insurance. Obviously, the hedged position consisting of the reference portfolio and a put option has a return $R_{H}$, which is a convex function of $R_{M}$ (Figure 8).

On the other hand, LELAND (1980) shows that in the limit any twice continuously differentiable convex payoff function $Y\left(R_{M}\right)$ can be generated by combining the reference portfolio, the riskless asset and put options. Therefore, any investment policy with a strictly convex payoff function is called a general portfolio insurance policy (Figure 9). LELAND (1980) shows how individual investors should deviate from the market portfolio.

In the following we explain general portfolio insurance in a slightly different framework.

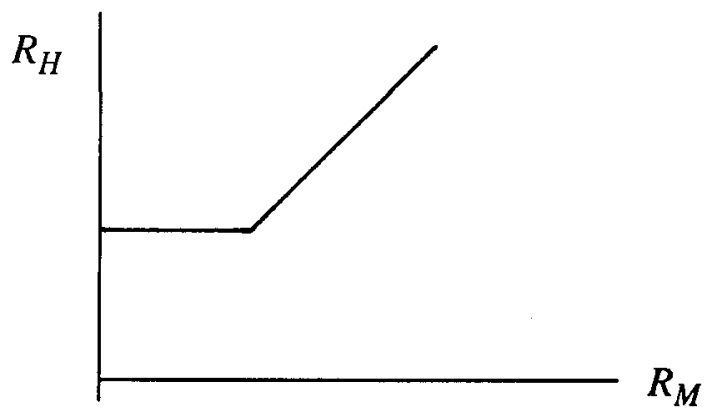

Figure 8 . 


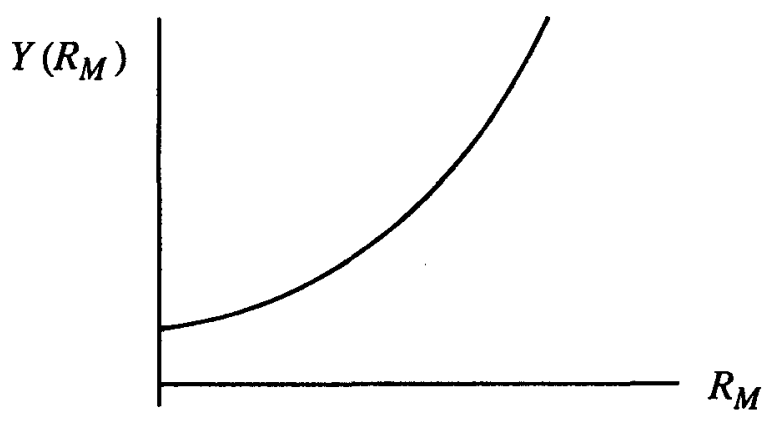

FigURE 9.

ASSUMPTIONS

C.1 There are

$m_{1}$ investors with NM utilities $u_{i} \in U(c), i=1, \ldots, m_{1}$

$m-m_{1}$ investors with NM utilities $u_{i} \in U\left(c^{\prime}\right)$, $i=m_{1}+1, \ldots, m$, where $c, c^{\prime} \in(0, \infty)$.

C.2 The initial wealth in the economy consists of $W_{0}$ units of the riskless asset and $W_{M}$ units of the reference portfolio.

According to C.2 total final wealth is given by the random variable $W_{0} R_{0}+W_{M} R_{M}{ }^{15}$ From Borch's theorem one sees immediately that all Pareto efficient allocations $\left(Y_{1}, \ldots, Y_{m}\right)$ are of the form

$$
\begin{aligned}
Y_{i} & =a_{i}^{1}+b_{i}^{1} X^{1}, & i & =1, \ldots, m_{1}, \\
Y_{i} & =a_{i}^{2}+b_{i}^{2} X^{2}, & i & =m_{1}+1, \ldots, m,
\end{aligned}
$$

with

$$
\begin{aligned}
a^{1}+a^{2} & =W_{0} R_{0}, \\
b^{1} X^{1}+b^{2} X^{2} & =W_{M} R_{M},
\end{aligned}
$$

where

$$
\begin{array}{ll}
a^{1}=\sum_{i=1}^{m} a_{i}^{1}, & a^{2}=\sum_{i=m_{1}+1}^{m} a_{i}^{2} \\
b^{1}=\sum_{i=1}^{m_{1}} b_{i}^{1}, & b^{2}=\sum_{i=m_{1}+1}^{m} b_{i}^{2} .
\end{array}
$$

Furthermore, according to Section 5.2 the following aggregation is possible:

Investors $i=1, \ldots, m_{1},\left(m_{1}+1, \ldots, m\right)$ can be represented by a single investor

15. $R_{0}$ and $R_{M}$ are considered here as exogenously given. 
with a NM utility $\hat{u}(\hat{\hat{u}})$. $\hat{u}$ and $\hat{\hat{u}}$ are characterized by

$$
\begin{aligned}
& \hat{u}^{\prime}(w)=(w-\hat{W})^{-c} \\
& \hat{u}^{\prime}(w)=(w-\hat{\hat{W}})^{-c^{\prime}} .
\end{aligned}
$$

Applying Borch's theorem once more leads to

$$
\frac{\left(a^{1}+b^{1} X^{1}-\hat{W}\right)^{-c}}{\left(a^{2}+b^{2} X^{2}-\hat{\hat{W}}\right)^{-c^{\prime}}}=\lambda
$$

with $\lambda>0$,

or by considering (32)

$$
\frac{\left(A^{1}+b^{1} X^{1}\right)^{-c}}{\left(A^{2}+W_{M} R_{M}-b^{1} X^{1}\right)^{-c^{\prime}}}=\lambda
$$

where

$$
\begin{aligned}
& A^{1}=a^{1}-\hat{W} \\
& A^{2}=a^{2}-\hat{\hat{W}} .
\end{aligned}
$$

Finally, one obtains

$$
W_{M} R_{M}=b^{1} X^{1}-A^{2}+\lambda^{1 / c^{\prime}}\left(A^{1}+b^{1} X^{1}\right)^{c^{\prime c}} .
$$

From (35) one concludes:

(1) $c=c^{\prime}: X^{1}$ is linear in $R^{M}$

(2) $c>c^{\prime}: X^{1}$ is concave in $R^{M}$

(3) $c<c^{\prime}: X^{1}$ is convex in $R^{M}$.

\section{INTERPRETATION}

1) For $c=c^{\prime}$ linear risk sharing is Pareto-efficient and no options are needed in the economy.

2) For $c<c^{\prime}$ investors $i=1, \ldots, m_{1}$, choose a general portfolio insurance policy. It is important to note that $c$ is not directly related to the absolute or relative risk aversion. ${ }^{16}$

\section{ACKNOWLEDGEMENTS}

The author is indebted to an anonymous referee for very helpful suggestions.

\section{REFERENCES}

BlACK, F. (1972). Capital market equilibrium with restricted borrowing, Journal of Business $\mathbf{4 5}$, 444-454.

BlaCK, F., JENSEN, M. C., and SCHOLES, M. (1972). The capital asset pricing model: some empirical tests, reprinted in M. C. Jensen, ed., Studies in the Theory of Capital Market. Praeger, New York, 1972, 79-124.

16. $c=-w u^{\prime \prime}(w) / u^{\prime}(w)$ holds only if $\hat{W}_{k}=0$ in (27a) and (27c). 
Blume, M. and Friend, I. (1973). A new look at the capital asset pricing model, Journal of Finance, March 1973, 19-34.

BORCH, K. (1960). The safety loading of insurance premiums, Skandinansk Aktuarietidskrift 43, 163-184.

CAss, D. and Stiglitz, J. E. (1970). The structure of investor preferences and asset returns, and separability in portfolio allocation: a contribution to the pure theory of mutual funds, Journal of Economic Theory 2, 122-160.

Copeland, T. E. and Weston, J. F. (1988). Financial Theory and Corporate Policy. AddisonWesley Publishing Company, third edition.

FAMA, E. F. and MACBETH, J. (1973). Risk, return and equilibrium: empirical test, Journal of Political Economy, May/June 1973, 607-635.

HAKANSSON, N. H. (1969). Risk disposition and the separation property in portfolio selection, Journal of Financial and Quantitative Analysis, 401-416.

Harrington, D. R. (1987). Modern Portfolio Theory, the Capital Asset Pricing Model and Arbitrage Pricing Theory: a user's guide. Prentice-Hall, Inc., second edition.

INGERSOLL, J. E. (1987). Theory of Financial Decision Making, Rowman \& Littlefield, New Jersey.

LELAND, H. E. (1980). Who should buy portfolio insurance?, Journal of Finance 35, May 1980, $581-594$.

LiNTNER, J. (1965). The valuation of risky assets and the selection of risky investments in stock portfolios and capital budgets. Review of Economics and Statistics, February 1965, 13-37.

Markowitz, H. (1952). Portfolio selection, Journal of Finance 7, 77-91.

Merton, R. C. (1982). On the microeconomic theory of investment under uncertainty. In Handbook of Mathematical Economics, Vol. II, North-Holland, Amsterdam.

Roll, R. (1977). A critique of the asset pricing theory's tests, Journal of Financial Economics, March 1977, 129-176.

Ross, S. A. (1978). Mutual fund separation in financial theory: the separating distributions, Journal of Economic Theory 17, 254-286.

SHANKEn, J. (1987). Multivariate proxies and asset pricing relations: living with the Roll critique, Journal of Financial Economics 18, 91-110.

Sharpe, W. F. (1963). A simplified model for portfolio analysis, Management Science, January $1963,277-293$.

SHARPE, W. F. (1964). Capital asset prices: a theory of market equilibrium under conditions of risk, Journal of Finance, September 1964, 425-442.

HEINZ MÜLLER

Institut für Empirische Wirtschaftsforschung, Universität Zürich, Kleinstrasse 15, CH-8008 Zürich, Switzerland. 